\title{
CFD Validation with LDV Test Data for Payload/Fairing Internal Flow
}

\author{
Max Kandula* \\ Sierra Lobo, Inc. (USTDC), Kennedy Space Center, FL 32899 \\ Khaled Hammad** \\ Dantec Dynamics Inc., 200 Williams Dr., Ramsey, NJ 07430 \\ Paul Schallhorn ${ }^{* *}$ \\ NASA Kennedy Space Center, FL 32899
}

\begin{abstract}
Flowfield testing of a $1 / 5^{\text {th }}$ scale model of a payload/fairing configuration, typical of an expendable launch vehicle, has been performed. Two-dimensional (planar) velocity measurements were carried out in four planes with the aid of Laser Doppler Velocimetry (LDV). Computational Fluid Dynamics (CFD) analysis results for the scale model flowfield are compared with the test data. The CFD results are in general agreement with the test data. The ability of the CFD methodology in identifying the global flow features (including critical points such as vortex, saddle point, etc.) has been demonstrated. Practical problems and difficulties associated with the LDV method applied to the complex geometry under consideration have been summarized.
\end{abstract}

\section{Nomenclature}

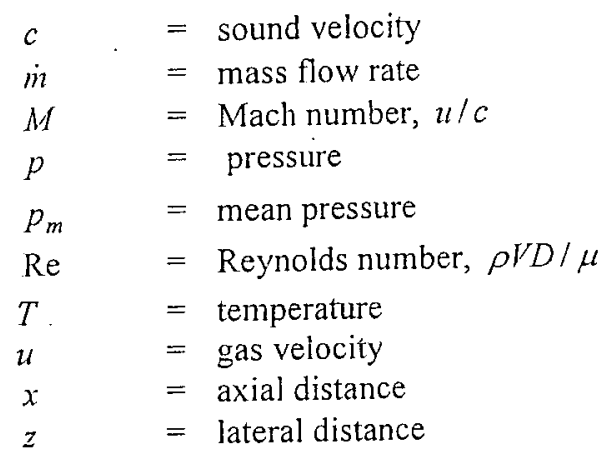

\section{GREEK SYMBOLS}

$$
\begin{array}{ll}
\mu & =\text { dynamic viscosity } \\
\rho & =\text { density }
\end{array}
$$

\section{SUBSCRIPTS}

$$
\begin{aligned}
f & =\text { full scale } \\
m & =\text { model } \\
\infty & =\text { ambient fluid }
\end{aligned}
$$

Sr. Principal Investigator, Mail Stop ASRC-3, Kennedy Space Center, FL 32899, Associate Fellow AIAA. ** Senior Application Specialist, Member AIAA

*** Thermal/Fluids Engineer, Launch Services Program, Sr. Member AIAA 


\section{Introduction}

$\mathrm{n}$ a recent article by Kandula and Walls ${ }^{1}$, the application of three dimensional Computational Fluid Dynamics (CFD) analysis to the internal flow of a payload/fairing pre-launch air cooling system, typical of an expendable launch vehicle (ELV), has been described and documented. The flow is turbulent and highly three-dimensional, and is relatively incompressible. It is characterized by vortex flow, swirl flow, separated flow, and boundary layer flow.

The CFD analysis of the above system was carried out with the aid of NASA OVERFLOW code (Buning et al. $^{2}$; Kandula and Buning ${ }^{3}$ ) in conjunction with an overset grid system ${ }^{4}$. Turbulence models implemented in this code include one-equation models of Baldwin-Barth ${ }^{5}$ and Spalart-Allmaras ${ }^{6}$. Two equation models such as Menter's SST-model (shear stress transport) ${ }^{7}$, and $k-\omega$ model (Kandula and Wilcox ${ }^{8}$ ) have also been incorporated and investigated.

The validity of CFD analysis is critically dependent on the choice of turbulence model. Two equation models provide better flow physics than one-equation models with regard to adverse pressure gradients, flow separation etc. However, for practical application involving three-dimensional flows, one equation model is frequently considered (ex. Space Shuttle ascent flowfield ${ }^{9}$ ), since two-equation models and higher order models are cost prohibitive despite their ability to include improved flow physics.

Majority of the validations of the OVERFLOW code with one-equation models have been carried out for external flows involving boundary layer flow's, and free shear layers, and mildly separated flows. Validation of the code for incompressible internal three-dimensional flows is very limited. Furthermore, the validity for strongly separated flows and flows with high degree of swirl has not been well established

For the payload/fairing application, a one-equation Spalart-Allmaras ${ }^{6}$ turbulence model was chosen. It solves for the turbulent kinetic energy distribution in the flowfield. In an effort to improve confidence in the CFD models for ELV fluid dynamics applications, a $1 / 5^{\text {th }}$ scale model of the payload/fairing was built, and the test data for the velocity field was correlated with CFD solutions. Gaseous nitrogen (GN2) was used as the test fluid. Laser Doppler Velocimetry (LDV) method was chosen for planar (two-dimensional) velocity measurements. This report summarizes the test facility, test data and CFD validation.

\section{Test Model Development}

\section{A CFD Geometric Model}

The CFD geometric model is the same as that considere 3 d in Ref. 1 . The overall grid system is comprised of 12 individual grids, amounting to a total of 2.5 million grid points. Prior to launch, cold air is delivered to the fairing through an air conditioning (AC) pipe, and flows past the spacecraft to provide adequate cooling for the spacecraft components. Major components of the encapsulated spacecraft model include the hexagonal main structure, ALI (Advanced land Imager), Hyperion, solar array (stowed), and X-band boom.

\section{B. Scaling Methodology}

The scaling methodology is based on the consideration that for dynamic similarity, the Reynolds number $R e$ in the full scale model (prototype) is the same as that in the scale model ${ }^{10}$. That is:

$$
R e_{f}=R e_{m}
$$

where

$$
\operatorname{Re}=\rho V D / \mu
$$

The geometric scale model is decided to be $1 / 5$. The flow parameters, such as velocity, mass flow rate, etc. in the prototype and the model are accordingly altered. During testing, difficulties with particle seeding with olive oil necessitated that the flow rate should be reduced by at least a factor of 4 . Thus model testing and CFD comparisons are performed at a reduced pipe Reynolds number of $0.6 \times 10^{5}$ corresponding to the reduced flow rate, while the full flow (or full scale) Reynolds number is $2.4 \times 10^{5}$. A summary of the scaling parameters for the full flow and the reduced flow is provided in Table 1. 


\section{Test Model Fabrication}

A photographic view of the $1 / 5^{\text {th }}$ scale model is presented in Fig. 1. The fairing model was made up of lexan material with a thickness of $0.06 \mathrm{in}$. Owing to the curvature of the fairing nose, the model nose was divided into several longitudinai sections to facilitate fabrication. The spacecraft model was made out of aluminum, and was painted black to minimize light reflections.

\section{Flow Loop}

Fig. 2 shows a schematic of the flow loop. The gaseous nitrogen (GN2) supply was obtained from a tube bank at 2400 psig capacity. The pressure is reduced by pressure regulators. A particle seeder (SCITEK LS-10 Remote Operation Liquid Droplet Seeder) was installed in the loop, providing olive oil spray required for LDV measurement. The seeder is operated by an independent air supply at 100 psig. A flexible hose ( 2 in. diameter) of about $14 \mathrm{ft}$. length was installed between the seeder outlet and the fairing model. The flexible hose serves to establish a fully developed flow, and to uniformly distribute the seeder particles before entering the test section. The test article is mounted vertically, with the bottom placed about $3 \mathrm{ft}$. above the ground level to minimize ground effects of the exiting flow. An oil pan is placed directly beneath the fairing to collect the oil in the exhaust stream.

Coriolis type mass flowmeter is employed to measure the GN2 mass flow rate. Pressure is measured by a differential pressure transducer. Temperature is measured by an RTD type transducer. All these instruments were calibrated prior to testing.

\section{LDV System}

\section{A. System Description}

Dantec Dynamics performed planar LDV measurements. LDV testing was conducted with backscatter-mode Fiberflow with a $60 \mathrm{~mm}$ probe and F80 processor. The focal length of the lens is $300 \mathrm{~mm}$. Spectra Physics model $177 \mathrm{CW}$ system with Ar-Ion laser was used. The laser power corresponds to: $514 \mathrm{~nm}, 60 \mathrm{~mW}, 0.7 \mathrm{~mm}$ diameter, 1.2 mrad beam divergence; and $486 \mathrm{~nm}, 60 \mathrm{~mW}, 0.7 \mathrm{~mm}$ diameter, $1.2 \mathrm{mrad}$ beam divergence. Each of the two colors is used for measuring one velocity component. The Burst Spectrum Analyzer (BSA) can provide up to more than 100,000 velocity measurements per sec.

The 3-D traverse has a range of $310 \mathrm{~mm} \times 310 \mathrm{~mm} \times 310 \mathrm{~mm}$. From the built-in software, a Cartesian mesh with the desired resolution is defined for each plane of measurement.

\section{B. Particle Seeding}

In the present experiments, olive oil was used as the seeding particle. The installation of the seeder and the flowmeter is seen in Fig. 3a. The SCITEK seeder, which is comprised of 10 Laskin nozzles producing air jets for atomization, was obtained from Dantec Dynamics. Droplets are generated by the shearing of the sonic jets. The droplet size is in expected to be in the range of 0.5-5 $\mu \mathrm{m}$, with mean droplet diameter roughly about 2 to $3 \mu \mathrm{m}$. As a rough guide, a $3 \mu m$ diameter oil droplet follows the fluid motion up to $1 \mathrm{kHz}$, and a $1 \mu m$ diameter particle up to $10 \mathrm{kHz}^{11}$. This estimate is based on Stokes law of resistance $\left(\right.$ Stokes ${ }^{12}$ ). Thus it is believed that the particles are able to follow the fluid motion. Typical photographic view of the laser beams is presented in Figs. $3 \mathrm{~b}$.

The overall error in LDV is believed to be about 2 percent, which includes random errors and systematic errors. The probable error and the maximum error in the measured velocity are estimated to be 3.8 percent and 5.5 percent respectively. Both these estimates include the LDV error of 2 percent.

\section{TV. Safety Precautions}

The test was conducted in a high bay building. Care was exercised in following the safety precautions as required by the safety office. Because of laser irradiation, laser training was obtained by all the personnel working in the test area. In addition, a laser eye examination and certification was provided. The test article was surrounded by an enclosure (weld curtains) on all sides in order to provide protection (prevent exposure) from the laser beams. Special safety goggles for the eyes were employed. Because of the nitrogen environment, an oxygen monitor was 
placed in the test area. The monitor produces an alarm if the oxygen level is below 19.8 percent. Dispersion of olive oil exiting the test section was also a safety concem. High pressure in the storage bottles is regulated to a much lower pressure levels inside the building. Proper ventilation from the droplet-laden exhaust from the model was also important.

\section{Test System Operation}

Problems were encountered on account of low particle seeding level and condensation of moist air on the outside of the model. At the nominal flow rate of $22 \mathrm{lbm} / \mathrm{min}$, the seeding was found to be inadequate as the sampling rate was unacceptably low. Also condensation of air on the outside of the faring model prevented the signal shortly after the test started. The condensation problem arose because of nitrogen expansion from the bottle pressure of about 2000 psig to about 2 psig near the model, thus reducing the nitrogen line temperature (to about 48 $\mathrm{F}$ to $50 \mathrm{~F}$ ) below the dew point of ambient air. A decision was therefore made to reduce the flow rate by a factor of 4 (Reynolds number is now reduced by a factor of 4 ). This afforded a good sampling rate of about $1000 \mathrm{samples} / \mathrm{sec}$, and also reduced the test time for a single plane from about 4.5 hours to about $1.5 \mathrm{hr}$. It also kept the oxygen concentrations to acceptable levels during each test run.

Thus, operation at the lower flow rate (Reynolds number) became necessary in view of the difficulties encountered with regard to seeding quality (olive oil), condensation, oil diffusion and deposition (accumulation), laser power, sampling rate, nitrogen atmosphere, test duration, closed doors (safety requirement), etc.

\section{Measurement Planes}

Test data acquisition was limited to four planes only because of the difficulty of measurements. Post test examination suggested that the measurements in two of the planes were of questionable character. Thus comparisons in the present report will be limited to two planes (plane-1 and plane-3), their locations being shown in Figs 4a and 4b. Planes 1 and 3 are coplanar, and pass through the axis of the fairing. Plane- 1 is located in the fairing nose region, while plane 3 is located between the pipe outlet and the solar panel. The master plane containing planes 1 and 3 bisects the solar panel, and is oriented at about $6 \mathrm{deg}$. from the pipe axis.

In the present measurements, the mesh length in either direction is in the ranges of $7 \mathrm{~mm}$ to $12 \mathrm{~mm}$. A total of about 300 points constitute each plane. At each measurement point, about 3000 data samples are considered in order to provide statistical confidence in the measured velocity.

\section{CFD and Test Comparisons}

As the testing was accomplished at $1 / 4^{\text {th }}$ the full scale Reynolds number, a CFD solution at the test (reduced) Reynolds number has been obtained with the OVERFLOW code. Steady state was achieved in about 4000 iterations. With the aid of TECPLOT software, the CFD solution was interpolated into the measurement planes where test data were obtained.

Figs. 5a and 5b show a comparison of the CFD and the test data in plane-1 corresponding to the fairing nose region. The CFD solution predicts three interesting critical points (2 vortex structures of opposite sign, and one saddle point). Critical points (or singular points) represent points in the flow where the velocity magnitude vanishes. They present topological information concerning the structure of the velocity vector field (or flowfield). It is apparent form Fig. $5 b$ that the measurements have captured only the saddle point, while the two vortices could not be measured because of the difficulty of measurements. The saddle point location ( $\mathbf{z}^{\prime}=-3$ in,, and $x=8$ in.) predicted by CFD agrees well with the test data.

The comparisons for plane-3 (region enclosed between the pipe outlet and the solar panel) are shown in Figs. 6a and $6 \mathrm{~b}$. Both the CFD and test data show two vortices. One well-organized vortex is seen below the level of the diffuser, while another vortex is observed above the diffuser. There is overall agreement for the bottom vortex (location and extent) predicted by the CFD agrees satisfactorily with that shown by the test data. There is seen greater offset in the location of the upper vortex. 


\section{Conclusion}

Although the LDV testing of the payload/fairing for the flowfield was shown to be feasible, it introduced a number of limitations and difficulties with regard to the scope and range of testing. These difficulties are related to particle seeding, air condensation and safety (including nitrogen environment). As a result, data could be gathered only at $1 / 4^{\text {th }}$ the full Reynoids number and in only a few planes. Nonetheless, the CFD solution was shown to capture important flowfield characteristics such as vortex motions. It is recommended that future testing should consider air instead of GN2 in order to alleviate problems concerning safety and condensation.

\section{Acknowledgments}

This work was supported by Launch Services Program (LSP) at Kennedy Space Center. Gary O'Neil of LSP contributed to the initial test planning and coordination. Thanks are due to Maria Littlefield of LSP for her continuing interest in this work.

\section{References}

${ }^{1}$ Kandula, M., and Walls, L., An application of overset grids to payload/fairing internal flow CFD analysis, AIAA2002-3253, 20 $0^{\text {th }}$ AIAA. Applied Aerodynamics Conference, St. Louis, Missouri, June 2002.

${ }^{2}$ Buning, P.G. et al., OVERFLOW users manual, Version 1.6s, NASA Langley Research Center, 1998.

3 Kandula, M., and. Buning, P.G., Implementation of LU-SGS algorithm and Roe upwinding scheme in OVERFLOW thin layer Navier-Stokes code, AIAA-94-2357, $25^{\text {th }}$ AIAA Fluid Dynamics Conference, Colorado Springs, CO, June 1994.

${ }^{4}$ Benek, J.A., Buning, P.G., and Steger, J.L., A 3-D grid embedding technique, AIAA-85-1523-CP, July 1985.

${ }^{5}$ Baldwin, B.S., and Barth, T.J., A one-equation turbulence transport model for high Reynolds number wallbounded flow's, NASA TM-102847, August 1990.

6 Spalart, P.R., and Allmaras, S.R., A one-equation turbulence model for aerodynamic flows, AIAA-92-0439, January 1992.

${ }^{7}$ Menter, F.R., Zonal two-equation $k-\omega$ turbulence models for aerodynamic flows, AIAA-93-2906, 1993.

${ }^{8}$ Kandula, M., and Wilcox, D.C., An examination of k-w turbulence model for boundary layers, free shear layers and separated flows, AIAA-95-2317,26 AIA A Fluid Dynamics Conference, San Diego, CA. June 1995.

${ }^{9}$ Slotnick, J.P., Kandula, M., and Buning, P.G., Navier-Stokes Simulation of Space Shuttle launch vehicle flight transonic flowfield using a large scale chimera grid system, AIAA-94-1860, 1994.

${ }^{10}$ Shames, I., Mechanics of Fluids, $2^{\text {nd }}$ edition, McGraw-Hill, New York, 1982.

${ }^{1}$ Dantec Dynamics, Laser Doppler Anemometry- Introduction to Principles and Application, 2004.

${ }^{12}$ Adrian, R.J., Laser Anemometry, in Fluid Mechanics Measurements (ed. R.J. Goldstein), Hemisphere, New York, pp. $155-240,1983$.

${ }^{13}$ Stokes, G.G., Camb. Phil. Soc., Vol. 9, part II, pp. 8-106, 1851. 
Table 1. Scaling Parameters

\begin{tabular}{|l|l|l|l|l|}
\hline Parameter & Full scale & $\begin{array}{l}\text { Full scale } \\
\text { Reduced Flow) }\end{array}$ & Scale Model & $\begin{array}{l}\text { Scale Model } \\
\text { (Reduced Flow) }\end{array}$ \\
\hline Geometric scale factor & 1 & & $1 / 5$ & $1 / 5$ \\
\hline Reynolds number & $2.4 \times 10^{5}$ & $0.593 \times 10^{5}$ & $2.4 \times 10^{5}$ & $0.593 \times 10^{5}$ \\
\hline Pipe inlet diameter, in. & 10 & 10 & 2 & 2 \\
\hline Inlet pressure, $\mathrm{psi}$ & 14.7 & 14.7 & 14.7 & 14.7 \\
\hline Inlet temperature, $\mathrm{F}$ & 58 & 58 & 55 & 55 \\
\hline Fluid & Air & Air & Nitrogen & Nitrogen \\
\hline Speed of sound, $\mathrm{ft} / \mathrm{s}$ & 1115 & 1115 & 1115 & 1115 \\
\hline Mass flow rate, $\mathrm{lbm} / \mathrm{s}$ & 1.851 & 0.463 & 0.370 & 0.0925 \\
\hline Vol. Flow rate, $\mathrm{ft} 3 / \mathrm{min}$ & 1450 & 362.5 & 291 & 72.75 \\
\hline Average velocity in pipe, $\mathrm{ft} / \mathrm{s}$ & 45.9 & 11.475 & 229 & 57.25 \\
\hline Mach number in pipe & 0.04 & 0.01 & 0.20 & 0.05 \\
\hline
\end{tabular}

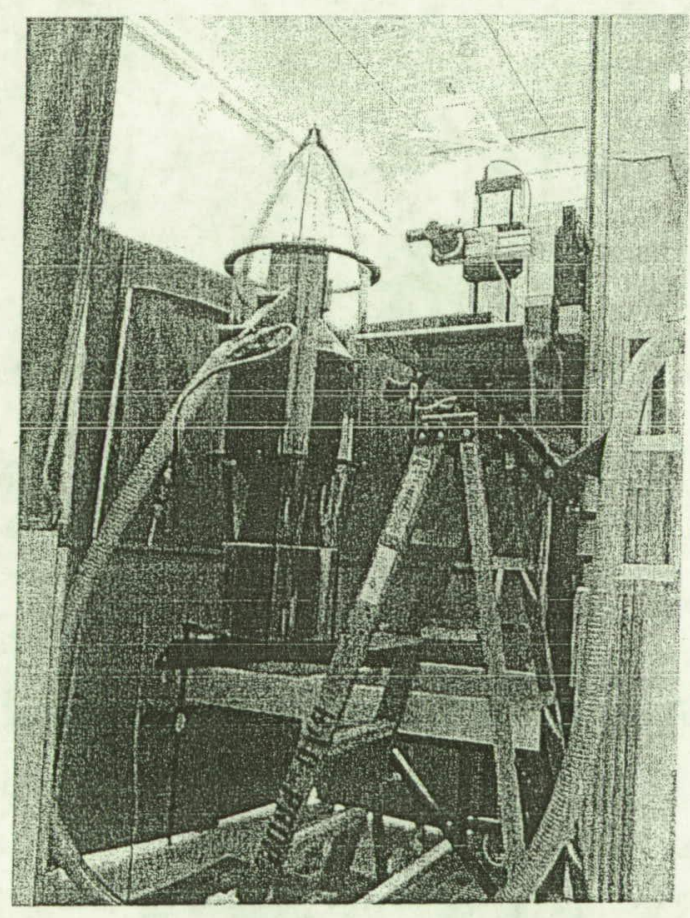

Figure 1. Photographic view of the test model. 


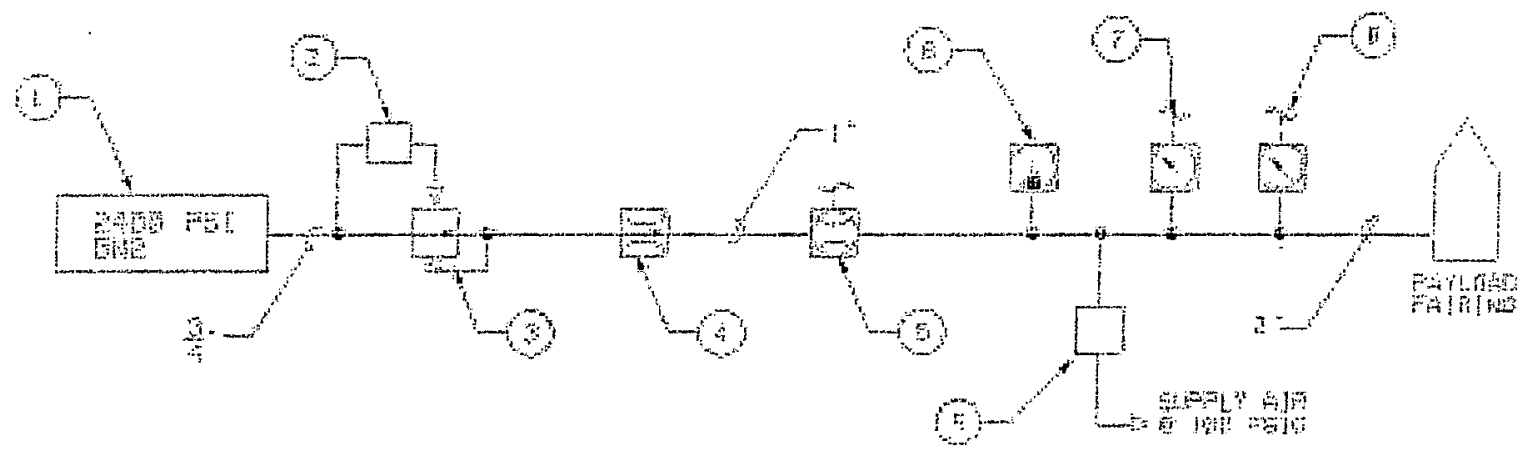

\begin{tabular}{|c|c|}
\hline Hen m, & noptrmen \\
\hline$i$ & 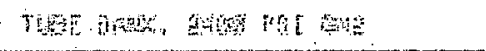 \\
\hline $\mathbb{Z}$ & Fonto \\
\hline$s$ & 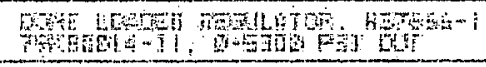 \\
\hline a & 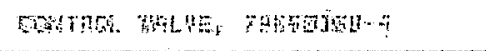 \\
\hline$\ddot{i}$ & 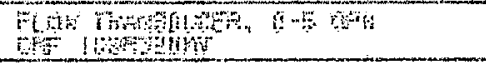 \\
\hline$\therefore$ & 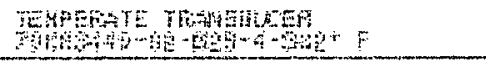 \\
\hline$\because$ & 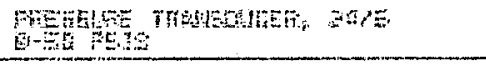 \\
\hline$a$ & 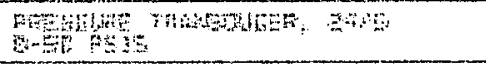 \\
\hline $\bar{i}$ & 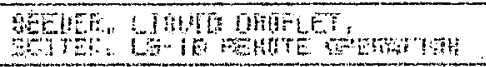 \\
\hline
\end{tabular}

Figure 2. Schematic of the flow loop. 


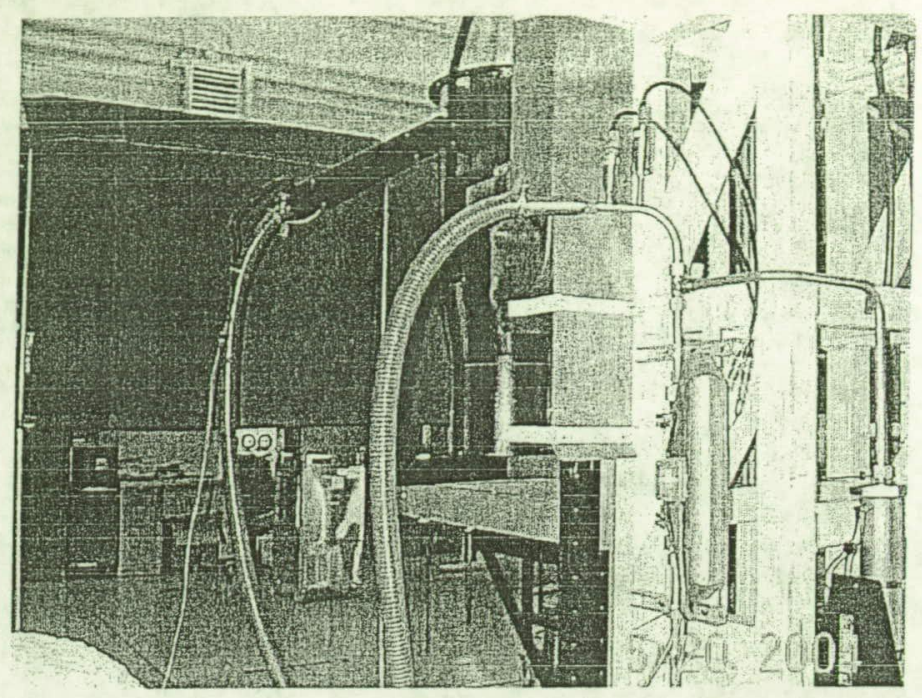

Figure 3a. Photographic view of the seeder and the flowmeter.

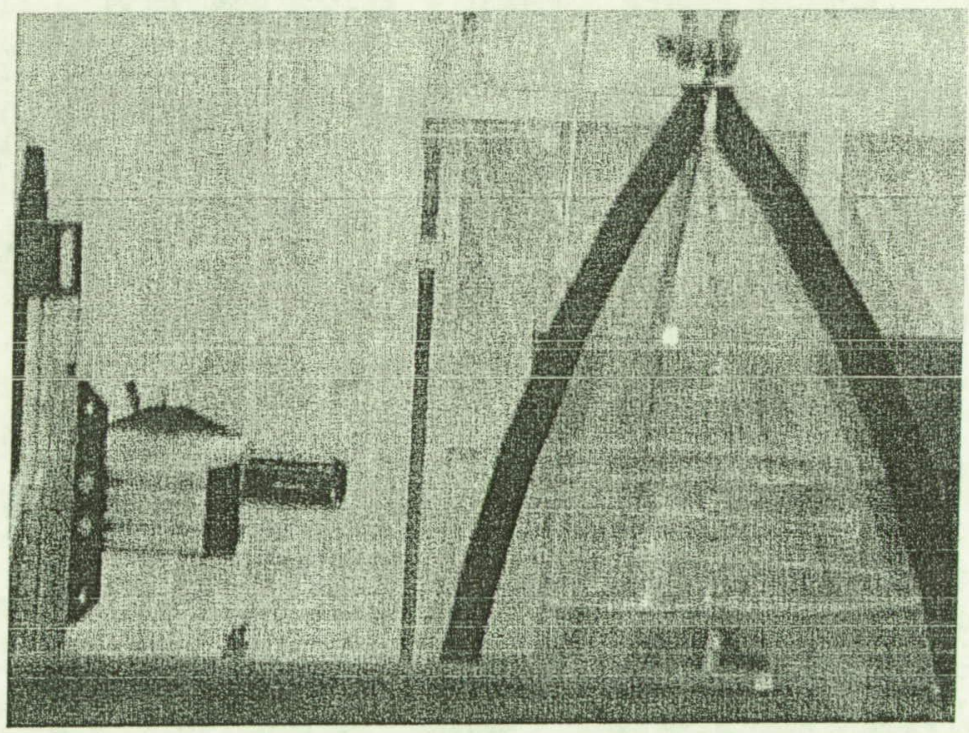

Figure 3b: Photographic view of the laser beams. 


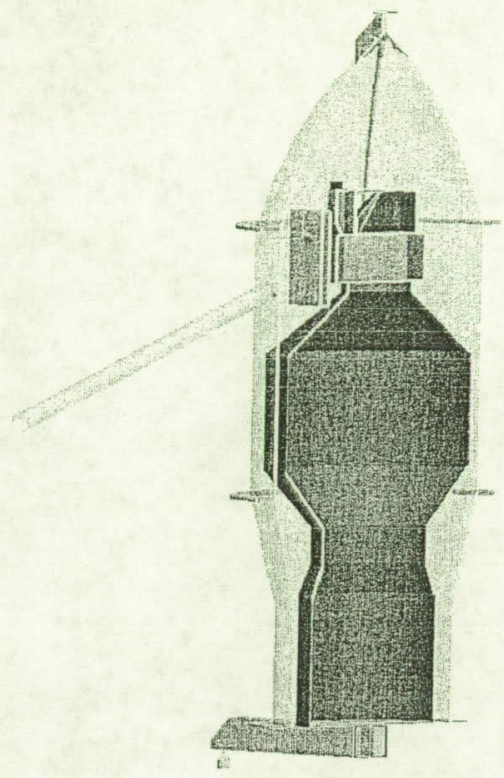

Figure 4a. Measurement plane iocations.

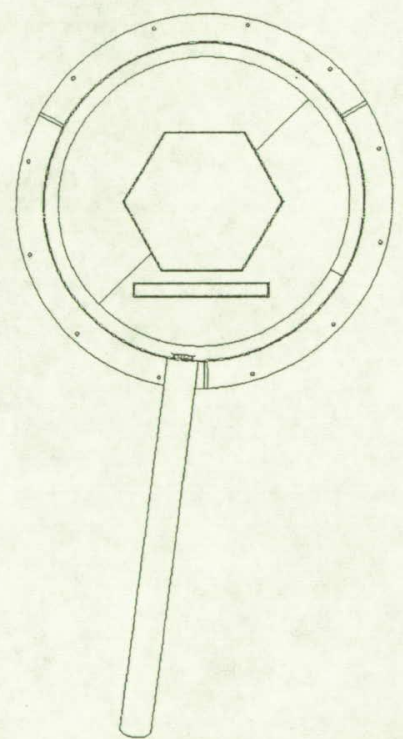

Figure 4b. Measurement plane Iocations (contd.). 


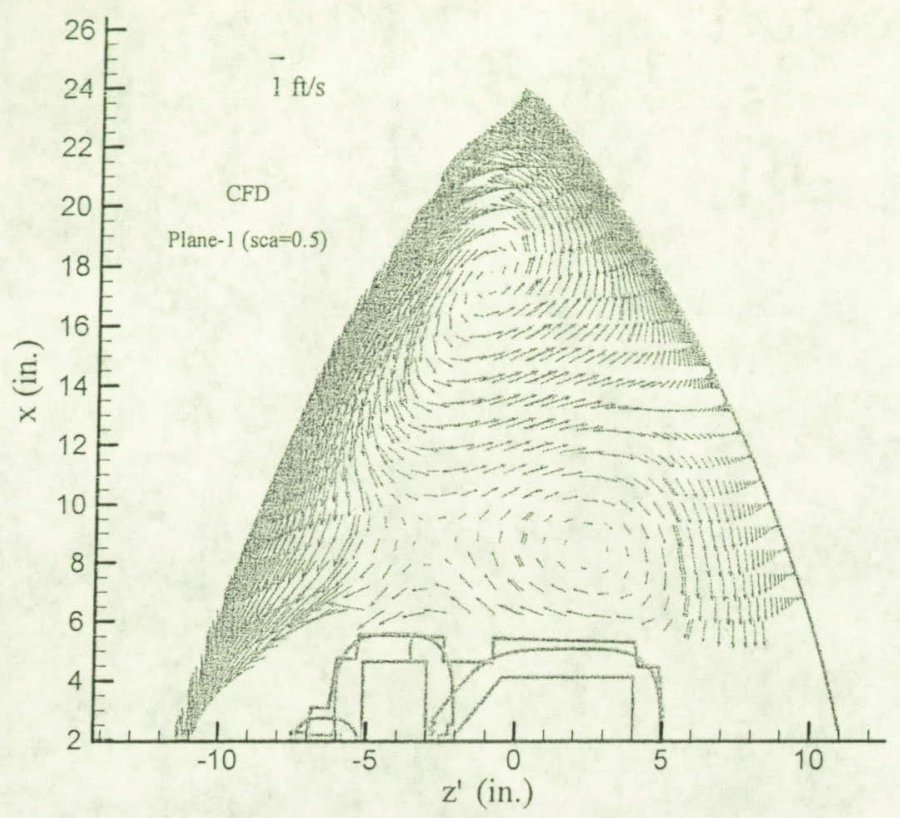

Figure 5a: Velocity vectors from CFD for plane-1.

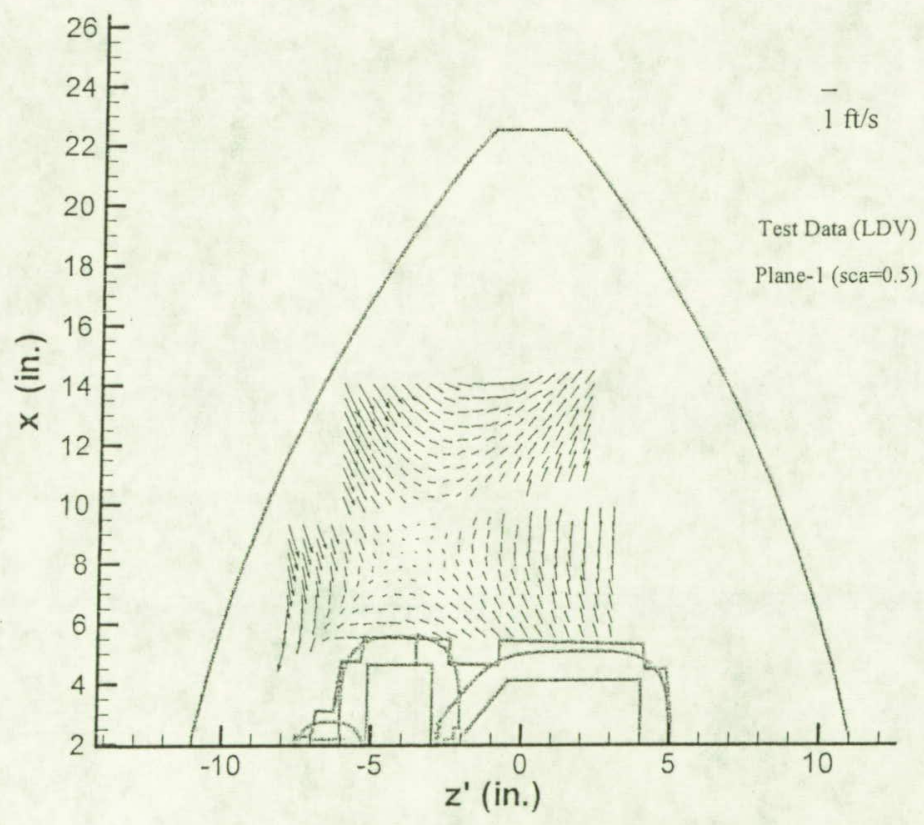

Figure 5b: Velocity vectors from test data for palne-1. 


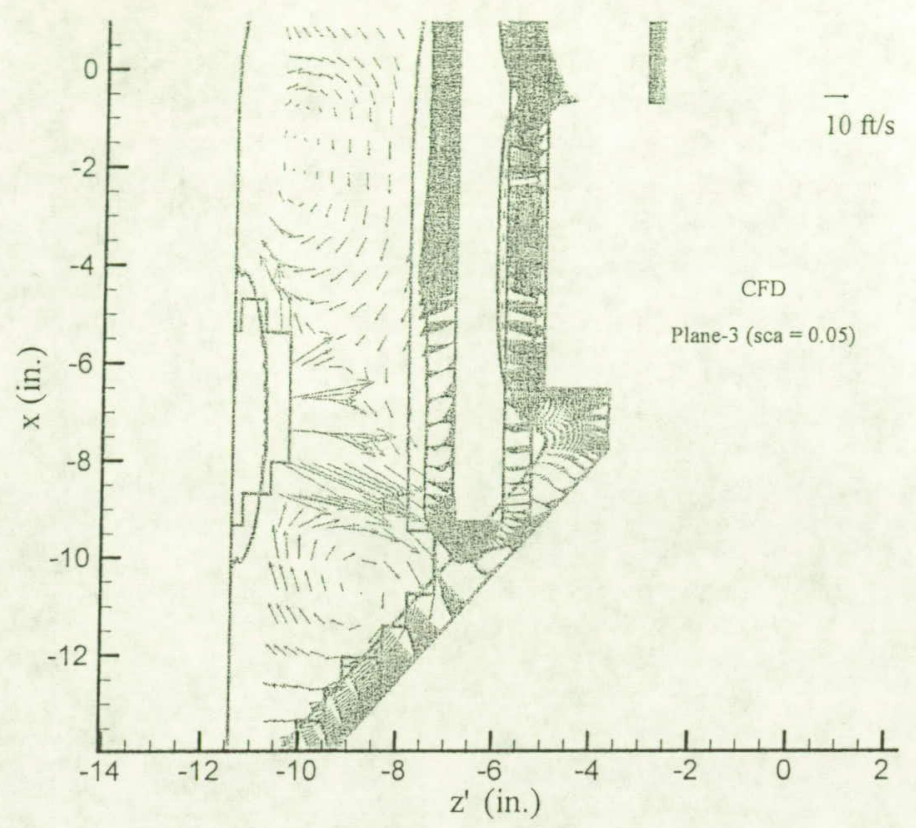

Figure 6a: Velocity vectors from CFD for plane-3

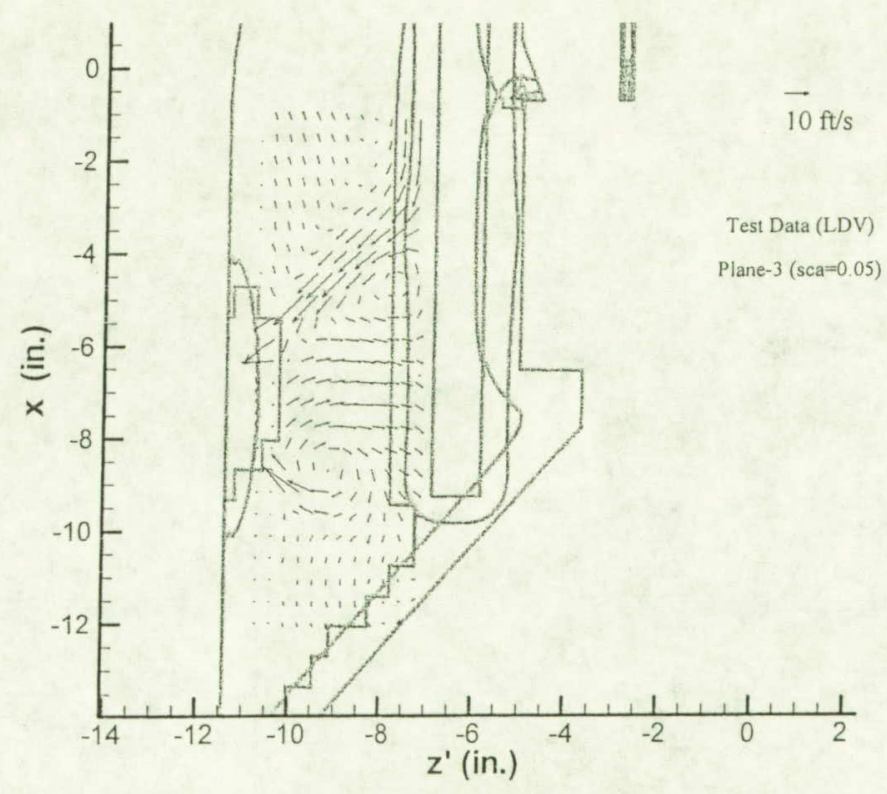

Figure 6b: Velocity vectors form test data for plane-3. 